BOURGES, L., «Calidad y seguridad alimentarias europeas: ¿lujo y necesidad o un enfoque global extendido?», REDUR I4, diciembre 20I6, págs. I7-29. ISSN I695-078X

\title{
CALIDAD Y SEGURIDAD ALIMENTARIAS EUROPEAS: ¿LUJO Y NECESIDAD O UN ENFOQUE GLOBAL EXTENDIDO?
}

\author{
Leticia BOURGES \\ DOCTORA EN DERECHO \\ SeCretaria General del Comité Europeo de Derecho Rural \\ UNIVERSITÉ PARIS I PANTHÉON-SORBONNE
}

SumARIO: I. Introducción. II. Calidad. II.r. Signos de calidad. III. Seguridad alimentaria y la PAC. III.I. Desperdicio alimentario: desafío y necesidad para la seguridad alimentaria. IV. Conclusiones

RESUMEN: El trabajo intenta reflexionar sobre tres temas dispares e interrelacionados: las cuestiones de calidad, la seguridad alimentaria y el nuevo desafío de gestionar las pérdidas y desperdicios alimenticios. Temas que parecen dispares terminan relacionándose y mostrando la necesidad de un enfoque integral para lograr un desarrollo equilibrado y sustentable. Las preocupaciones sobre la calidad sanitaria de los productos agrícolas termino incluyendo los agricultores en la cadena alimenticia y ahora la preocupación por las pérdidas y desperdicios parece querer incluir a los consumidores.

PAlabras Clave: Calidad alimentaria, calidad productos agrícolas, signos de calidad, seguridad alimentaria, residuos alimentarios, pérdida de alimentos

ABSTRACT: The paper tends to reflex on three different and interrelated issues: quality questions, food security and the new challenge of food waste and loss management. Issues that seem to be different end to be related and show the need to have an integral approach in order to reach a balanced and sustainable development. Concerns on food security of agricultural products make farmers to be considered part of the food chain supply and now the preoccupations related to losses and waste seems to aim to include consumers.

KEYWORDS: Key Words: food quality, agricultural products quality, signs of quality, food security, food waste, food loss 


\section{Introducción}

Las cuestiones de calidad y de seguridad alimentaria se insertan perfectamente en el nuevo enfoque integral con el que se aborda la agricultura y que se adopta a nivel económico ${ }^{\mathrm{I}}$.

El enfoque integral que se impone a la agricultura puede asegurarse sólo en cuanto se incentiven el intercambio de información ${ }^{2}$ y el uso de la tecnología para lograr un desarrollo durable ${ }^{3}$. En esta perspectiva han influido la preocupación por temas ambientales, las crisis de sanidad y seguridad alimentarias, la volatilidad de los precios de las materias primas, las preocupaciones de los consumidores, el desarrollo de los derechos humanos y de las mujeres, así como las consideraciones respecto de la familia agrícola, y la preocupación de la comunidad internacional sobre los resultados de recientes investigaciones ${ }^{4}$ y los eventos acaecidos en relación al cambio climático ${ }^{5}$ (que requieren también una acción a nivel local).

Dentro de todas esas cuestiones y factores que han ido modificando la manera de abordar la agricultura, las cuestiones de calidad y los signos de calidad tienen una relevancia especial no solo por responder a las cuestiones sanitarias sino en cuanto son vistos como instrumentos útiles para responder a otras cuestiones, como contribuir a la viabilidad de las explotaciones y a la generación de externalidades positivas económicas y sociales de la agricultura.

Las cuestiones relativas a la seguridad sanitaria alimentaria si bien se han ido reglamentando en la medida que se presentaban, buscan evitar todo riesgo para la salud asumiendo un claro antropocentrismo, ubicando a la salud como un valor superior y elevando la figura del consumidor.

Este antropocentrismo se refleja en la vigilancia de la seguridad alimentaria, que suma la cuestión ambiental y la humana, y pretende equilibrar el resguardo del ambiente y la necesidad de los alimentos. Circunstancia ésta que pone ahora el foco en el consumidor atribuyéndole la responsabilidad de hacer atención respecto de los desperdicios alimentarios.

\footnotetext{
I Comision, Comunicación Cerrar el círculo: un plan de acción de la UE para la economía circular, $\operatorname{COM}(2015) 6 I 4$ final.

${ }^{2}$ La información es vital para asegurar usar los métodos de producción más convenientes adaptados al medioambiente local. Es recomendable incorporar el conocimiento tradicional/autóctono - muy común en áreas rurales y en algunos países -y proteger ese conocimiento de la apropiación indebida usando instrumentos del derecho de propiedad intelectual. Una gestión efectiva del conocimiento tradicional, los recursos genéticos y las innovaciones locales debe ser organizado.

3 El texto de negociación de París (FCCC/20I5/ADP/I) tiene un enfoque de abajo hacia arriba. Los objetivos individuales pueden fortalecer los compromisos de los estados gradualmente.

4 IPCC $5^{\text {th }}$ Assessment Report (AR5).

5 V. Sendai Framework for Disaster Risk Reduction 2015-2030, Third UN World Conference on Disaster Risk Reduction, Sendai, I4-I8 March 20I5, A/CONF.224/.2 (UNISDR). UNICEF, The Challenges of Climate Change: Children on the front line, 2014.
} 
Nos proponemos abordar someramente el esquema de calidad de los productos agroalimentarios (II) con el particular aporte de los signos de calidad (II.I) y la preocupación actual por el resguardo de la seguridad alimentaria a través de protección de la capacidad productiva amenazada por el cambio climático (III) con el desafío de la lucha contra los desperdicios alimentarios (III.I).

\section{Calidad}

La definición de calidad ha sido preferentemente obviada por la carga subjetiva que en general implica. Sin embargo, tanto científica como legalmente se ha tratado de circunscribirla a través de diferentes instrumentos.

Los trabajos conducidos en el XVII Congreso del CEDR, concluyeron una constatación similar: tal definición constituiría una tarea imposible ya que existen sólo representaciones de calidad, variables histórica y geográficamente. Un análisis de derecho comparado demostró que «dos elementos parecen comunes a todos los países: I) la calidad umbral/entrada al mercado, bajo la cual un alimento deja de ser seguro y arriesga fuertemente de alterar la salud del consumidor, 2) la calidad convencional, definida por el contrato y los usos». Sin embargo, entre esos trabajos se distinguía la perspectiva de los países mediterráneos que a esas acepciones «agregan la calidad organoléptica, gustativa o estética que hace que un producto sea 'de calidad'».

El Libro Verde sobre la calidad de los productos agrícolas (COM(2008) 64I final) señaló que la calidad implica responder a las expectativas del consumidor, es decir que hace referencia a las características de los productos -métodos de producción incluidos- « que los agricultores desean hacer conocer mejor y que los consumidores desean conocer ». Por eso, se considera que la calidad es un conjunto de características que responden a las obligaciones, sean convencionales o legales, y que se traducen en un signo de calidad o una mención determinada.

En el sector agroalimentario, existen dos tipos de calidades que tienen que ver con la existencia misma del producto en el mercado. La primera es la calidad sanitaria, que constituye la calidad umbral que permite su existencia mercantil -el producto puede ser introducido en el mercado solamente en cuanto cumpla con ese mínimo de requisitos establecidos por las reglas de higiene que hacen de él un producto sano para el consumo. Las reglas de higiene trascienden la calidad sanitaria del producto en cuanto se refieren a todo lo concerniente a su manipulación y el control correspondiente ${ }^{6}$, y se completan con aquellas que se refieren a los límites máximos de tolerancia de aditivos o de ciertas sustancias nocivas -como los pesticidas. Superado la calidad umbral que determina el «producto sano», el producto debe responder a un siguiente nivel de calidad: la calidad nominativa. Las nominaciones comerciales en cuestión son determinadas por la ley, que establece las características que debe tener un producto para poder ostentar la

\footnotetext{
6 V. los reglamentos que constituyen lo que se dio a llamar el «paquete higiene» $(852 / 2004 / C E$, 853/2004/CE, 854/2004/CE y 882/2004/CE, y la directiva 2002/99/CE).
} 
denominación pertinente . Esta «calidad» hace a la circulación comercial del producto. Así, esa nominación es un símbolo de calidad en la medida que es capaz de comunicarle al consumidor las características de un producto. Las denominaciones legales son determinantes para la comercialización de un producto y tienen fuertes connotaciones jurídicas y económicas tanto en los ámbitos del consumo -por la información que transmite y la repercusión en la relación del consumidor con el producto- como de la competencia -por el valor económico que tiene el respeto de las características que implica una cierta denominación. En la UE (art.5, par.I, dir. 2000/13/CE) la denominación legal o de comercialización de un producto alimentario es aquella prevista por las disposiciones comunitarias que le sean aplicables, en su defecto es aquella prevista por las disposiciones nacionales del país donde el producto sea vendido, y sino el nombre consagrado por los usos (nominación usual) del Estado miembro en el cual se efectúe la venta, o en último recurso, una descripción del producto o, si es necesario, de su utilización suficientemente precisa para permitir al comprador de conocer la real naturaleza y de distinguirlo de productos con los cuales pudiera haber lugar a confusión (nombre descriptivo -art. 2, parr. 2, al. « $\mathrm{n}$ », règl.II69/20II/UE). La nominación utilizada en el Estado miembro de producción es válida en el de comercialización si el producto es legalmente fabricado y comercializado en el primero, si el producto que designa no se distingue demasiado, sea desde el punto de vista de su composición como de su fabricación, del producto conocido bajo esa nominación en el segundo; y si las indicaciones en el etiquetado alcanzan para asegurar una información correcta para los consumidores del Estado miembro de la comercialización ${ }^{8}$.

Con el fin de satisfacer otras demandas, el sector agroalimentario ha distinguido otro tipo de calidades dando lugar a signos distintivos de calidad con el fin de satisfacer otras demandas: poner de relevancia las características organolépticas generalmente ligadas al origen geográfico, la revalorización de la zona de producción específica o vulnerable (zonas de montaña), evidenciar los métodos de producción (agricultura orgánica o biológica) o el respeto de las tradiciones o de los métodos comerciales (comercio equitable).

La Unión europea ha tenido un rol importante en este desarrollo alentando la alta calidad atribuida a productos con profunda tradición cultural. La política de calidad se ha

\footnotetext{
7 V.: CJ (III cám.) del I4 julio I988, proced. de redressement judiciaire c/Smanor SA, aff. C-298/87, Yaourt surgelé, Rec. I988 p. 04489 . CJ, sentencia del 9 dic. I98I, Comisión c/Rép. italiana, Vinaigre I, aff.ı93/80, Rec. ı98I page 030I9. CJ (V cám.), sentencia del I4 julio ı994, proced. penal c/ J.J.J. Van der Veldt, aff. C-I7/93, Rec. I994 p. I-0537.

Dir. 2000/36/CE del Parlamento europeo y del Consejo del 23 junio 2000 relativa los productos de cacao y de chocolate destinados a la alimentación humana, DO L I97 du 3.8.2000, p. I9. Regl. (CE) nI82/2009 de la Comisión del 6 marzo 2009 modificando el reglamento (CE) nIoI9/2002 relativo a las normas de comercialización del aceite de oliva, DO L 63 du 7 marzo 2009, p.6-8.

${ }^{8}$ V.: CJ, sentencia del 20 febrero 1979, Cassis de Dijon, aff. ı20/78, Rec. I979, p. 00649. CJ (V cám.), sentencia del I4 julio I994, proced. penal c/ J.J.J. Van der Veldt, aff. C-I7/93, Rec. I994 p. I-0537. CJ (II cám.), sentencia del I9 febrero I98I, proced. penal c/ Fabriek voor Hoogwaardige Voedingsprodukten Kelderman BV, aff. I30/80, Rec. I98I p. 00527. CJ (II cám.), sentencia del I7 marzo I983, proced. penal c/ De Kikvorsch Groothander-Import-Export Bv, Bières, aff. 94/82, Rec. I983 p. 00947 . CJ, sentencia del 9 dic. I98I, Comisión c/ Rép. italiana, Vinaigre I, aff.ı93/80, Rec. I98I p. 03019. CJ, sentencia del I5 octubre I985, Comisión c/ Rép. italiana, Vinaigre II, aff. 28I/83, Rec. I985 p. 03397.
} 
convertido en un elemento esencial de las medidas de desarrollo rural, y particularmente de zonas rurales desfavorecidas 9 . En ese trabajo y política se distinguen los signos de calidad que en cierta manera tratan de proteger el distintivo patrimonio de producción alimentaria europeo.

\section{II.I. Signos de calidad}

Los países mediterráneos son proclives a identificar la calidad con la calidad organoléptica de los productos y para ello han fomentado el sistema de signos de calidad en vistas a valorizar su producción auténtica y la preservación de costumbres. Los primeros reglamentos sobre el tema fueron fundamentalmente fomentados por Francia y por ello llevaban la fecha del I4 de julio. La actual normativa es el reglamento (UE) nII5I/20I2 sobre los regímenes de calidad de productos agrícolas y alimenticios, al cual se llegó luego de un largo proceso -en el 20 Io se presentó el «Paquete calidad» ${ }^{\mathrm{IO}}$, seguido de una consulta pública ${ }^{\text {II }}$ cuyos resultados se reflejan en la Comunicación sobre la política de calidad de los productos agrícolas ${ }^{\mathrm{I} 2}$ que establece los principios estratégicos. La refundación de los sistemas de calidad busca la simplificación de los procedimientos y de la reglamentación abarcando las denominaciones de origen protegidas (DOP), las indicaciones geográficas protegidas (IGP), las especialidades tradicionales garantizadas (ETG), la producción biológica, y el marco legal para establecer algunas indicaciones facultativas. Los esquemas de calidad abordados por el reglamento abarcan los productos agrícolas y agroalimentarios, los vinos, las vinos aromáticos y las bebidas espiritosas, los productos de agricultura biológica, los venidos de regiones remotas (DOM franceses, y Azores) y de zonas de montaña ${ }^{\mathrm{I} 3}$.

La mencionada reforma de la reglamentación de los signos de calidad no es anodina y se inserta en las líneas económicas más amplias de la UE, que busca incentivar la

9 Cf. CEDR, L'agriculture multifonctionnelle, Aspects juridiques, L’Harmattan, Paris, I999, notamment : Hudault, J., Rapport de synthèse, p.7i6 et s.

io Proposición de la Comisión de reglamento del Parlamento europeo y del Consejo sobre los esquemas de calidad de productos agrícolas, $\operatorname{COM}(2010) 733$ final, SEC(20I0)I524, Io diciembre 20Io. Proposición de la Comisión de reglamento del Parlamento europeo y del Consejo para enmendar el reglamento (CE) nº I234/2007 sobre normas de comercialización, COM(20I0)738 final -esta propuesta tenía el objetivo de establecer normas de comercialización básicas para todos los productos agrícolas y la posibilidad de adoptar normas sectoriales, como sobre la indicación del lugar de producción. Comunicación de la Comisión, Directrices sobre el etiquetado de los productos alimenticios que utilizan como ingredientes DOP e IGP, DOUE/20IO/C34I, I6.I2.20I0 p.3-4. Comunicación de la Comisión, Directrices de la UE sobre las mejores prácticas aplicables a los regímenes voluntarios de certificación de productos agrícolas y alimenticios, DOUE20I0/C34I, I6.I2.20Io p.5-II.

http://ec.europa.eu/agriculture/quality/policy/quality-package-2oIo/index_en.htm

II Lanzada por el Green Paper on Quality (COM(2008)64I).

I2 28 mayo 2009, (COM(2009)234). V. también: Report Agricultural product quality: what strategy to follow, 28 marzo 20I0, a iniciativa del Parlamento europeo. Comunicacion de la Comision: The CAP towards 2020: meeting the food, natural resources and territorial challenges of the future. Res. del Parlam. 23 junio 20I,I sobre dicha comunicación.

I3 Regl. delegado (UE) n665/20I4 de la Comisión, del it de marzo 20I4, que completa el regl. (UE) $\mathrm{n}^{\circ} \mathrm{II} 4 / 20 \mathrm{I2}$ en lo que atañe las condiciones de utilización del término de calidad facultativo «producto de montaña», DO LI79 de I9.6.20I4, p.23. 
generación de valor agregado para aumentar la competitividad de las explotaciones agrícolas y favorecer la diversificación de la agricultura, manteniendo como objetivo el desarrollo durable de las zonas rurales. En este sentido la política de calidad es un elemento clave y estratégico de la política agrícola ${ }^{\mathrm{T}}$, pues este tipo de productos tienen una fuerte conexión con el territorio.

Se ha tratado de mejorar la protección creando un marco más robusto imponiendo una obligación de protección ex officio por parte de los Estados miembros, exigiéndoles la adopción de instrumentos públicos de $\operatorname{control}^{\mathrm{I}}{ }^{5}$. Estos controles quedan ligados a la cooperación interestatal en materia de protección de consumidores ${ }^{16}$. Además, se acentúa la promoción de la calidad de los productos. A pesar de ello, la reforma ha sido criticada por no haber tenido más audacia y haber innovado más ${ }^{17}$.

Sin embargo, no todos los productos están sometidos a signos de calidad, y cuando se habla de seguridad alimentaria es dable incluir a todos y contemplar reglas para asegurar su disponibilidad.

\section{Seguridad alimentaria y la PAC}

Conviene aclarar que en este segmento nos referimos a la seguridad alimentaria en tanto food security, es decir a la disponibilidad de los alimentos en términos cuantitativos a fin de que sean suficientes para satisfacer las necesidades nutricionales y energéticas del consumidor $^{\mathrm{I} 8}$. La seguridad alimentaria es un concepto complejo. Fragmentariamente podemos analizar su objeto, el producto -es decir la cantidad de productos adecuados, sanos y aceptables (subjetivamente determinado por el consumidor) disponibles- o los medios utilizados para concretar la disponibilidad, sea a través de canales comerciales o a través de la producción -en el sentido de productividad y capacidad productiva. Mientras en la primera perspectiva entran a jugar términos económicos y comerciales, en la segunda han surgido nociones como la soberanía alimentaria o la autosuficiencia alimentaria.

Es claro que las políticas públicas tienen un papel decisivo en la concreción de la seguridad alimentaria, pues la disponibilidad de los productos luego exige ser acompañada

I4 V.: Parlam. Resol. la agricultura como sector estratégico en el contexto de la seguridad alimentaria (DO C I36 E de II.5.20I2, p. 8)

Is Se ha considerado que es una respuesta al caso Parmesan y la necesidad de resguardar la unicidad de la protección de naturaleza pública acordada por el reglamento. Los Estados miembros también están obligados por la directiva 2006/29/CE sobre las prácticas comerciales desleales (de II de mayo de 2005, DO LI49, II.06.2005, p.22).

${ }^{16}$ Regl. (CE) n.2006/2004 del Parlamento y del Consejo, del 27 de octubre de 2004, sobre la cooperación entre las autoridades nacionales encargadas de la aplicación de la legislación de protección de los consumidores, DO L364, 9.I2.2004, p.I.

Una clara protección de los consumidores es la disposición de indicar el porcentaje del producto que goza de signo de calidad, cuando éste es utilizado como ingrediente y por tanto como una ventaja comparativa del producto en cuestión. V. d.lgs. 297/04, Italia.

I7 N. Coppola, Regolamento II5I/2OI2 su DOP, IGP ed STG: una occasione mancata?, in Alimenta Vol. XXI, 2OI3, n. 2, 3I.

I8 Plan d'action du Sommet mondial de l'alimentation, I996, www.fao.org. 
por la posibilidad de acceso a los productos, situación que involucra otras políticas públicas que contribuyen a poner en situación al ciudadano a estar en condiciones de acceder al alimento (transporte, políticas macro-económicas, sociales, etc.).

La Unión europea ha tenido como objetivo histórico asegurar la provisión de alimentos sanos de alta calidad, nutritivos y a precios abordables (art.39 TFUE). La seguridad alimentaria abordada desde su perspectiva objetiva -es decir a partir de su objeto mismo, el producto alimenticio- se cristaliza en la disponibilidad de productos adecuados en cantidades suficientes y aceptables. La sanidad del producto, a los efectos de asegurar la adecuación del mismo, ha debido ser abordada por la legislación. Las diferentes crisis sanitarias han hecho evolucionar la legislación adoptando un enfoque que abarca toda la cadena de provisión alimentaria «de la granja a la mesa» ${ }^{19}$, mejorando los sistemas de control y de reacción y sometiéndola a una vigilancia permanente. Los standards impuestos y las exigencias previstas (incluidos el etiquetado y las alegaciones) intentan asegurar la producción de alimentos en ese sentido.

Si nos concentramos en los medios para alcanzar la seguridad alimentaria de un Estado, éstos están limitados al comercio y a la propia producción. La política comercial es importante en tanto que puede afectar la producción misma y así la estructura agrícola del país.

Al analizar la seguridad alimentaria de un Estado, si bien es importante la producción, creemos que lo es también referirse a su capacidad productiva, pues ambas no son sinónimas y para el objetivo en cuestión es necesario que las políticas pertinentes se concentren en preservar la capacidad productiva del país más que fomentar la producción. Se trata pues de adoptar una perspectiva a largo plazo que se encuadre en el concepto de desarrollo sustentable actual. En los albores de este siglo, se ha debido enfrentar o tomar en cuenta riesgos que atacan mayormente la seguridad alimentaria en términos de productividad y de capacidad productiva. Estos riesgos y desafíos incluyen el cambio climático, la escasez de energía y recursos, la eventual disminución de productividad agrícola, el incremento de concentración en la cadena de provisión alimentaria, la volatilidad de precios, nuevos riesgos o resistencias bacteriológicas, la gestión de residuos; a los que se les atribuye la potencialidad de causar graves problemas de producción y de ingresos agrícolas (determinantes de los planes de producción). Por ello, en la actualidad se trata de difundir e incentivar las prácticas denominadas agro-ecología/agricultura-climainteligente (climate smart agriculture) ${ }^{20} \mathrm{o}$ intensificación ecológica ${ }^{2 \mathrm{I}}$, que se basan en el

I9 V. : Comisión, Livre blanc sur la sécurité alimentaire, COM(99)7I9 final, del I2 enero 2000. Regl. (CE) $\mathrm{n}^{\circ} \mathrm{I7} 8 / 2002$ del Parlam. y del Consejo del 28 enero 2002 estableciendo los principios generales y las prescripciones generales de la legislación alimentaria, instituyendo la Autoridad europea de la seguridad de los alimentos y fijando los procedimientos relativos a la seguridad de los alimentos, DO L3I del ${ }^{\text {ro }}$ de febrero 2002, p.I-24.

${ }^{20}$ FAO define así la agricultura climática inteligente: «Climate-smart agriculture (CSA) is an integrative approach to address the interlinked challenges of food security and climate change, that explicitly aims for three objectives: (I) sustainably increasing agricultural productivity, to support equitable increases in farm incomes, food security and development; (2) adapting and building resilience of agricultural and food security systems to climate change at multiple levels; and (3) reducing greenhouse gas emissions from agriculture (including crops, livestock and sheries). CSA invites to consider these three objectives together at different scales - from farm to landscape -at different levels- from local to global -and over short and long time horizons, taking into account 
respeto de la biodiversidad, el trabajo «con» la naturaleza, la rotación de cultivos, la utilización racional del agua y de otros recursos en una perspectiva de responsabilidad social, que requiere la asunción de las políticas y reglamentos apropiados para la práctica de esa agricultura, mientras se busca el aumento de la productividad y la capacidad de mitigación contra los riesgos climáticos, tratando de asegurar al mismo tiempo la viabilidad de las explotaciones y la generación de externalidades positivas económicas y sociales de la agricultura.

Ante un escenario con diversos riesgos y tipos de exigencias, en la Unión europea, la Comisión ha elaborado un documento sobre los escenarios futuros, hacia el año 2050 , proyectando las eventuales respuestas que deberían preverse. El documento ${ }^{22}$ ha identificado el impacto actual y los desafíos sobre el marco legislativo, así como la evolución futura de los mismos.

Se ha considerado que es vital la existencia de medidas transversales y de coherencia entre las distintas políticas, aunque éstas tengan particularidades propias. Son necesarias investigaciones y políticas para guiar los comportamientos tanto de los productores como de los consumidores. La agricultura recobra la prioridad productiva marcada de manera general por la sustentabilidad y se impulsan políticas alimentarias con campañas informativas respecto a adoptar una dieta sana y a evitar el derroche o despilfarro alimentario. La Comisión distingue también la necesidad de avanzar sobre una gobernanza

national and local specificities and priorities»y especifica que «what is new about CSA is an explicit consideration of climatic risks that are happening more rapidly and with greater intensity than in the past. New climate risks require changes in agricultural technologies and approaches to improve the lives of those still locked in food insecurity and poverty and to prevent the loss of gains already achieved. CSA approaches entail greater investment in: I- Managing climate risks, 2- understanding and planning for adaptive transitions that may be needed, for example into new farming systems or livelihoods, and 3- exploiting opportunities for reducing or removing greenhouse gas emissions where feasible» http://www.fao.org/climatechange/ climatesmart/en/. CGIAR Research Program on Climate Change, Agriculture and Food Security (CCAFS), Climate-smart agriculture, Success Stories, https://cgspace.cgiar.org/bitstream/handle/10568/34042/ Climate_smart_farming_successesWEB.pdf?sequence $=5$

La cuestión es mejorar las tecnologías, metodologías y enfoques para la gestión sostenible de la finca / métodos de producción. Agroecología es fuertemente reforzada por el Ministro francés de Agricultura. Véase la Conferencia de la UNESCO, julio de 20I5. Véase: Action contre la Faim, Rapport, Ensemble, créons un climat contre la faim (http://faimetclimat.com/docs/4PAGES_Climat.pdf) et Call from Civil Society organizations to fight hunger and climate change (http://faimetclimat.com/appel.pdf), 2015.

${ }^{21}$ Véase: R. Bommarco, D. Kleijn, S.G. Potts, Ecological intensification: harnessing ecosystem services for food security, Trends in Ecology \& Evolution, Vol.28, Issue 4, April 2013, pages 230-238. T. Doré, D. Makowski, E. Malézieux, N. Munier-Jolain, M. Tchamitchian, P. Tittonell, Facing up to the paradigm of ecological intensification in agronomy: Revisiting methods, concepts and knowledge, European Journal of Agronomy, Vol.34, Issue 4, May 20II, pages I97-210. Colloque Utilisation du potentiel biologique des sols, un atout pour la production agricole (in http://www.academie-agriculture.fr/colloques/utilisation-du-potentiel-biologique-dessols-un-atout-pour-la-production-agricole) y Agroforesterie tempérée et tropicale (http://www.academieagriculture.fr/colloques/agroforesterie-temperee-et-tropicale), Académie de l'agriculture de France, 20I5.

22 DGSanco, Scoping study, Delivering on EU food safety and nutrition in 2050 -Scenarios of future change and policy responses, Final Report, 20/12/2013.

DGSanco, Foresight Project for future food policy development, 20I5, presentado en conferencia en la Expo Universal Milano20I5: Safety and Nutrition in 2050, el I7 de julio de 2015. http://ec.europa.eu/dgs/health_food-safety/information_sources/events/20I507I7_safety_nutrition_en.htm

Documento previo de referencia: Scoping study, Delivering on EU food safety and nutrition in $2050-$ Scenarios of future change and policy responses, Final Report, 20/12/2013. 
internacional de la cadena de provisión alimentaria, de aplicar tecnología e innovación en el sistema alimentario sustentable, y de promover la diversidad en el sistema alimentario para poder sobrellevar cualquier irregularidad.

La reforma de la PAC de $2013^{23}$ se hizo eco de esa perspectiva profundizando las medidas ambientales, previendo la necesidad de fomentar la investigación, la transferencia de conocimientos y la innovación (partenariados europeos para la innovación; v. regl. Regl.1307/2013), e imponiendo el consejo profesional ${ }^{24}$.

En el primer pilar ${ }^{25}$, se han instaurado los pagos directos verdes y se simplifican y refuerzan las exigencias reglamentarias básicas que determinan la eco-condicionalidad de las ayudas y que se refieren a materia de medio ambiente, cambio climático, buenas condiciones agrarias y medioambientales de la tierra, salud pública, fitosanidad, sanidad y bienestar animal. El objetivo de las reglas que determinan la eco-condicionalidad ${ }^{26}$ de las ayudas tiene por objetivo contribuir al desarrollo de una agricultura sostenible y responder a las expectativas sociales.

El segundo pilar, enfocado en el desarrollo rural ${ }^{27}$, también ha sufrido una fuerte «verdización» respondiendo a la preocupación preponderante de proteger la capacidad productiva.

Es de destacar que la actividad forestal entra plenamente en consideración en estas previsiones $^{28}$ (art. 2I y s. regl. 1305/2013), pues es complementaria a la agricultura y elemento fundamental para alcanzar los objetivos horizontales de carácter ambiental o relacionados con el cambio climático.

Las medidas de desarrollo rural dan prioridad a la gestión sostenible y eficiente de recursos (la gestión del agua cobra un cierto protagonismo) ${ }^{29}$ y de ecosistemas, sosteniendo

23 El marco reglamentario está formado por el reglamento del Consejo y del Parlamento 1307/2013, completado por el reglamento de la Comisión delegado 639/20I4 y de ejecución 6I4/20I4 y el reglamento delegado ıоог/20I4. Las reglas horizontales -IACS, condicionalidad, sanciones, etc.- están establecidas por el reglamento del Consejo y del Parlamento I306/2013, completado por el reglamento de la Comisión delegado 640/20I4 y el de ejecución 809/20I4 y el 834/20I4.

24 Reglamento (UE) 1306/20I3 del Parlamento Europeo y del Consejo de I7 de diciembre de 2013 sobre la financiación, gestión y seguimiento de la Política Agrícola Común, DO L 347, 20.12.2013, p.549.

25 El reglamento del Consejo y del Parlamento I308/20I4 trata la organización común de mercado.

${ }_{26}^{6}$ Reglamento (UE) 1306/20I3 del Parlamento Europeo y del Consejo de I7 de diciembre de 2013 sobre la financiación, gestión y seguimiento de la Política Agrícola Común, DO L 347, 20.I2.2013, p.549, art.91 y s.

27 Las reglas para los programas de desarrollo rural están establecidas en el reglamento del Consejo y del Parlamento I305/20I3, seguido por el reglamento de la Comisión delegado 807/20I4 y el de ejecución 808/20I4. Como lo mencionan los considerandos del reglamento, la política de desarrollo rural debe integrar los objetivos estratégicos enunciados en la Comunicación de la Comisión Europa 2020 (3 marzo 20120), y ser coherente con los objetivos generales de cohesión económica y social establecidos en el TFUE.

${ }^{28}$ Regl. I305/20I3, art.2I y s. El art. 34 especifica las medidas en relación a los servicios silvoambientales y climáticos y la conservación de bosques.

29 Regl.I305/2013, art. I7 especifica que las ayudas para inversiones en activos físicos abarca inversiones materiales o inmateriales que mejoren el rendimiento global y la sostenibilidad de la explotación agrícola, o que se destinen a infraestructuras destinadas al desarrollo, modernización o adaptación de la agricultura y el sector forestal, o que no productivas estén vinculadas a la realización de objetivos agroambientales y en materia de clima. 
la transición hacia una economía a baja emisión de $\mathrm{CO}_{2}$ y resistente a los cambios climáticos, destinando a ellas un mínimo de $30 \%$ del paquete. La protección de los recursos implica preservar la capacidad productiva y al mismo tiempo se reconoce la necesidad de mantener la viabilidad y competitividad de las explotaciones agrícolas ${ }^{30}$. Todas las regiones entran en el ámbito de aplicación, pues la atención a las más afectadas por diferentes tipos de limitaciones responde a una concepción global. Además, en línea con esta perspectiva (económico-ambiental), se promueven los mercados locales y los circuitos cortos (glolocal).

En términos generales las medidas del segundo pilar son financiadas por un sobre nacional, por cofinanciamiento (entre 3\% y $85 \%$ según la región y las derogaciones en alta); siendo el 30\% del financiamiento FEADER (Fondo Europeo Agrícola de Desarrollo Rural) atribuido a medidas ambientales o climáticas. Una cuestión importante es que se prevé una mayor flexibilidad entre los dos pilares, permitiendo transferir de un pilar a otro (3billones de euros en 6 años a rever en 20I7).

III.I. Desperdicio alimentario: desafío y necesidad para la seguridad alimentaria

Es claro que la gestión de la seguridad alimentaria tiene un talón de Aquiles relativamente reversible en la pérdida y el desperdicio alimentarios. A partir del reglamento I78/2000/CE, que estableció las bases de la legislación alimentaria, se adoptó un enfoque global reconociéndose la responsabilidad de todos los agentes de la cadena alimentaria. En la actualidad, preocupa la cantidad de alimentos que terminan en los residuos mientras paralelamente hay personas que sufren deficiencia alimentaria, aun en países «ricos» ${ }^{\text {I }}$. La perspectiva global adoptada para regular la producción debe también adoptarse para regular esta faiblesse que afecta directamente la seguridad alimentaria, tanto en el aspecto de la capacidad productiva como de la disponibilidad de alimentos.

Ese enfoque global involucra ahora también al consumidor. La educación, la información y formación se han convertido en elementos importantes para evitar el fenómeno (desperdicio de alimentos consumibles) y solucionar un problema (carencia alimentaria). Por ello se considera que todo ambiente y medio es bueno para hacerle

30 Así es que el art. I8 del reglamento 1305/2013 se refiere a las medidas que tienden a la reconstitución del potencial de producción agrícola dañado por desastres naturales y catástrofes y la implantación de medidas preventivas adecuadas. El art. 38 prevé la subvención de fondos mutuales que se constituyan para paliar adversidades climáticas, enfermedades de animales y vegetales, infestaciones por plagas e incidentes medioambientales. Las cuestiones agroambientales y referidas al clima son objeto de medidas especificas en tanto se focalicen en el mantenimiento o a la promoción de cambios necesarios en las prácticas agrícolas que contribuyan positivamente al medio ambiente y al clima. El art. 29 se refiere al fomento a las prácticas y métodos de agricultura ecológica. Finalmente, encausado en la profundización de la innovación y el transferimiento de conocimiento, se fomenta la Asociación europea para la innovación en materia de productividad y sostenibilidad (art.55) con el fin de contribuir a alcanzar los objetivos de la Estrategia Europa 2020 de un crecimiento inteligente, sostenible e integrador.

${ }^{3}$ FAO: un tercio de la producción alimentaria destinada al consumo humano en el mundo es perdida o desperdiciada (equivalente as,300 millones de toneladas/año) mientras soo० millones de personas sufren hambre (http://www.fao.org/food-loss-and-food-waste/fr/). La preocupación también se hizo notoria en la Unión europea (Comisión europea, a.a., Preparatory Study on Food Waste Across EU27, 2010). El 20I4 fue declarado año contra el desperdicio alimentario. 
comprender a los consumidores que es inaceptable desechar inútilmente comida que podría haber servido ${ }^{32}$.

Sin dudas, el formar contra el desperdicio implica formar en una buena nutrición. En el marco de la política alimentaria europea se ha tratado de fomentar una mejor nutrición ${ }^{33}$. Una de las razones de este efecto es la constatación que cinco de los siete principales factores de riesgo de muerte prematura (hipertensión, colesterol, índice de masa corporal, ingesta inadecuada de frutas y hortalizas y abuso de alcohol) están vinculados a la alimentación.

Un factor crucial en el desperdicio alimentario es la apariencia (calidad visual) de los productos no transformados, de ahí que ello ha dado lugar a diversas campañas comerciales pero que podrían descubrir un nicho de mercado destinado a sanear el problema de acceso a los alimentos de personas con bajos recursos. Se trata de utilizar instrumentos de marketing para informar sobre la ausencia de diferencia cualitativa de un producto que tiene defectos de forma, en otras palabras, se trata de una especie de rehabilitación de frutas y verduras imperfectas o antiestéticas o no calibradas o que no entran en los standards ${ }^{34}$.

Cabe aclarar que hay que distinguir la pérdida del desperdicio ${ }^{35}$, en cuanto la primera se produce a lo largo de toda la cadena alimentaria (sobre todo en la etapa de almacenamiento) -siendo a veces inevitable- mientras el segundo se presenta sobre todo en el punto de distribución y de consumo. Por su parte, la directiva 2008/98/CE ${ }^{36}$, define residuo como cualquier sustancia u objeto del cual su poseedor se desprenda o tenga la intención o la obligación de desprenderse; y considera «biorresiduo» a los residuos alimenticios y de cocina procedentes de hogares, restaurantes, servicios de restauración colectiva y establecimientos de consumo al por menor, y residuos comparables procedentes de plantas de transformación de alimentos. Estos biorresiduos son tratables por las legislaciones y las políticas de prevención y gestión de residuos que abarcan diversas acciones (prevención, preparación para la utilización, reciclado, valorización y eliminación),

${ }^{2}$ Un elemento importante es la cuestión de la buena lectura del etiquetado y saber diferenciar data limite de utilización optimal (DLUO), data limite de consumo (DLC) -que es de respeto imperativo. Aunque igualmente es reconocido que se deben tener en cuenta otros detalles que caracterizan al producto o al embalaje y respetar el principio de precaución cuando de eventual ingesta se trata.

33 V. Plataforma europea de acción sobre alimentación, actividad física y salud (http://ec.europa.eu/health/nutrition_physical_activity/platform/index_es.htm); Grupo de alto nivel sobre alimentación y actividad física (http://ec.europa.eu/health/nutrition_physical_activity/high_level_group/index_es.htm); European Food Information Council (http://www.eufic.org/index/es/); Plataforma europea sobre la dieta y la actividad física (http://www.eufic.org/article/es/show/eu-initiatives/rid/plataforma-europea-accion-dieta-actividad-fisica/). Para diversas iniciativas respecto a la dieta v.: http://www.eufic.org/block/es/show/eu-initiatives/

34 En Francia: marca colectiva Gueules cassés; Les fruits et légumes moches (Intermarché v. https://youtu.be/lMARwAXmn_o) con un 30\% de reducción en el precio. En Canada: Second life -donner une seconde vie aux fruits et légumes moches (http://www.second-life.ca/). En el Reino Unido, el Waste and Resources Action Programme está dirigido a aquellos que aprecian la comida y detestan el desperdicio, registró una disminución del I3\% en los desechos sobre un periodo de tres años (2006/7-2010). Alineándose a los tiempos modernos, también se han desarrollado aplicaciones al respecto (Checkfood - http://checkfood.fr/).

35 V. FAO. Global food losses and food waste-Extent, causes and prevention, Roma, $201 \mathrm{~s}$.

${ }^{6} 6$ Dir. 2008/98/CE del Parlamento y del Consejo de i9 de noviembre de 2008 sobre los residuos, DO L3I2 del 2I.II.2008, p.3. 
debiéndose estimular las opciones de tratamiento que generen el mejor resultado medioambiental global, respetando los principios de precaución y sostenibilidad. Sin embargo, se prevée que estos residuos se destinen al compostaje, la digestión y la producción de materiales ambientalmente seguros.

El tema es importante no solo porque cuanto se tira, en alguna parte -aun cercanahay gente que sufre carencia alimentaria, sino también por el impacto ambiental ${ }^{37}$ que genera la producción (transformación y distribución incluidas) de ese alimento que no cumple su destino final (alimentar). Por tanto, el desperdicio alimentario genera un efecto social negativo (la persistencia de un derecho a la alimentación insatisfecho), e implica una pérdida económica y un impacto ambiental negativo ineficaz (sin cumplir su fin). En otras palabras, todo alimento tiene un costo económico y ambiental y un fin social (alimentar), si ese fin finalmente no se cumple, los costos se pierden y los recursos naturales se han visto afectados inútilmente.

El Parlamento europeo ${ }^{38}$ se ha comprometido con la cuestión, reclamando a la Comisión de elaborar acciones concretas destinadas a reducir a la mitad del desperdicio alimentario para el 2025, evitando su producción.

En Francia, en el 2013 se firmó el Pacto nacional contra el desperdicio alimentario que comporta ir medidas que comprometen a todos los actores de la cadena alimentaria. Luego se estableció el i 6 de octubre - día mundial de la alimentación- como día nacional de lucha contra el desperdicio alimentario. Un informe parlamentario (autoría de Guillaume Garot) fue presentado en abril del 20I5. El 2016 se anuncia con programa ambicioso: la instalación de un grupo de trabajo para evaluar las evoluciones reglamentarias sobre las fechas de caducidad y otro para intercambiar sobre la educación a la lucha contra el desperdicio alimentario y para proponer instrumentos pedagógicos adaptados, el ofrecimiento de una convención tipo para las donaciones de las superficies medianas y grandes a las asociaciones caritativas, la elaboración de guías de buenas prácticas para luchar contra el desperdicio alimentario para centros hospitalarios y medico-sociales, lanzamiento de una campaña de sensibilización. El 20I6 ha comenzado con la ley contra el desperdicio alimentario39, aprobada a la unanimidad, que prohíbe a la gran distribución de desechar los productos alimenticios invendidos. Establece una jerarquía de acciones para recuperar y valorizar los alimentos y evitar el desperdicio. Se estima que en Francia se desechan io millones de toneladas de alimentos por año y que cada francés hace lo mismo en razón de 20 a $30 \mathrm{~kg}$ por año ${ }^{40}$. Las superficies medianas y grandes de más de $400 \mathrm{~m} 2$ tendrán la obligación de concluir una convención con una asociación caritativa a fin de facilitar las donaciones alimentarias.

37 V. FAO, Empreinte des gaspillages alimentaires, Rome, $20 \mathrm{I} 2$; Id., Food wastage footprint, 2013.

${ }_{3}^{8}$ Parlement, résolution du i9 janvier 20I2 sur le thème « Eviter le gaspillage des denrées alimentaires : stratégies pour une chaîne alimentaire plus efficace dans l'Union européenne (20II/2I75(INI)). En línea con la Agenda 2030 para el desarrollo sustentable, (ONU, AG, A/70/I, 25 sept. 2015).

39 Ley n²0I6-I3 8 del in de febrero relativa a la lucha contra el desperdicio alimentario, JORF nº036 del I2 de febrero 2016.

4 Infografía en http://agriculture.gouv.fr/infographie-le-gaspillage-alimentaire-en-france 


\section{Conclusiones}

Las cuestiones ambientales, las relacionadas a la seguridad alimentaria y los parámetros económico-sociales han conducido a la idea de una economía circular ${ }^{4 \mathrm{I}}$. La multifuncionalidad de la agricultura a la que contribuyen los productos agroalimentarios bajo signo de calidad ha ya marcado un poco el camino. La cuestión del desperdicio alimentario viene ahora a marcar otro hito en la evolución de la concepción socioeconómica global de la cadena alimentaria.

Las crisis sanitarias introdujeron el enfoque global que impuso tener en perspectiva toda la cadena alimentaria. La problemática del cambio climático, del aumento de la población mundial y de la necesidad de cuidar la seguridad alimentaria, imponen ahora una perspectiva similar en relación a la alimentación. Dentro de la seguridad alimentaria, estudios determinaron la realidad y la consecuencia inútilmente nefasta del desperdicio alimentario. Se trata, como ha recomendado el Parlamento europeo, de tomar medidas para disminuir o evitar el desperdicio superfluo. Esto necesitará de normas que obliguen a la acción como la ley francesa, pero también a la estipulación de programas para educar sea a los agentes económicos como a los consumidores. Se necesitan definiciones claras distinguiendo la pérdida del desperdicio, pero quizás también incentivos para la etapa preventiva y fomentos para disminuir o eliminar los desechos evitables.

El consumidor no debe quedar ajeno, pues un gran porcentaje de los desperdicios alimentarios tienen su fuente en el consumidor y en este tema, hay una cuestión de responsabilidad social y ciudadana que deberá asumir. Si las demandas del consumidor concluyeron en signos de calidad, ahora, profundizando el carácter antropocentrista, se exige de él una contrapartida y un comportamiento responsable por el bien social de la humanidad.

${ }^{4}$ La Comisión europea se propone un plan de acción ambicioso con el fin de reforzar la competitividad, crear empleo y generar un crecimiento durable (dic. 20I5): Paquet sur l'économie circulaire. V.: Comision, Comunicación Cerrar el círculo: un plan de acción de la UE para la economía circular, COM(20I5)6I4 final. Respecto al desperdicio alimentario se espera adoptar medidas para reducirlo y un método común de medida, mejorar las practicas en materia de indicación de la data de consumo, adoptar instrumentos para alcanzar el objetivo mundial de desarrollo durable disminuyendo a la mitad los desperdicios alimentarios para el 2030; además el paquete incluye una proposición legislativa sobre residuos (COM(20I5)/595 final). 